\section{Relationship between skipping breakfast and impaired fasting glucose along with cardiovascular and pre-diabetes condition risk factors in apparently healthy subjects}

\author{
Yulan Li, 1 Tohru Nemoto, 1 \\ Satoshi Tobimatsu, 1 Masafumi Saito, ${ }^{1}$ \\ Midori Ebata, ${ }^{1}$ Hiromi Munakata,2 \\ Kei Nakajima1,2
}

1Division of Clinical Nutrition, Department of Medical Dietetics, Faculty of

Pharmaceutical Sciences, Josai University; 2Department of Internal Medicine, Social Insurance Omiya General Hospital, Bonsai, Kita, Saitama, Japan

\section{Abstract}

Regular food intake plays a pivotal role in normal glucose homeostasis. However, few studies have evaluated the level of fasting glucose in individuals with skipping breakfast, which theoretically means lack of supplementary energy and increased risk of subsequent hypoglycemia. We examined the prevalence of suspected habitual skipping breakfast (SHSB) (skipping at least three times /week), roughly assessed with a simple question, and fasting plasma glucose level, cardiovascular risk factors, and lifestyle factors in a cross-sectional study of 2,331 asymptomatic adults who had never been treated with insulin or oral medications for diabetes. The overall prevalence of SHSB was $16.3 \%$ (men $20.1 \%$, women $9.4 \%$, $\mathrm{P}<0.0001, \chi^{2}$ test). Compared with normal fasting glucose, impaired fasting glucose (IFG) (100-125 mg/dl), but not high fasting glucose ( $\geq 126 \mathrm{mg} / \mathrm{dL}$ ), was significantly associated with SHSB and this association remained after adjustment for relevant confounders [Odds Ratio (95\% CI): 1.75 (1.33-2.30) and 2.10 (0.934.71), respectively]. Age (inversely), current smoking, late dinner before sleeping, infrequent exercise, and high C-reactive protein ( $\geq 1.8 \mathrm{mg} / \mathrm{L}$ ) were simultaneously associated with SHSB independently of each other. In a subgroup of subjects who underwent a $75 \mathrm{~g}$ oral glucose tolerance test $(n=1,315)$ of pre-diabetes groups, isolated IFG $(\mathrm{n}=272)$ was only significantly associated with SHSB. Current results suggest that IFG, subtle inflammatory state, and unfavorable lifestyle factors may be associated with the habit of skipping breakfast in asymptomatic adults. Causality and detailed mechanisms remain to be clarified in further studies.

\section{Introduction}

Non-healthy eating behaviors, which include skipping breakfast, a late dinner, and an evening snack, have been considered to be unfavorable lifestyles, especially in public health. Of these, skipping breakfast is commonly observed among children as well as adolescents. ${ }^{1-3}$ However, skipping breakfast is not rare among adults, ${ }^{4-6}$ although the prevalence is lower than in children and adolescents in many cross-sectional studies.,2,5 In many population studies on adults, habitual skipping of breakfast was associated with other unfavorable lifestyle factors, such as smoking, infrequent exercise, drinking alcohol, and high energy intake, ${ }^{1-7}$ and with cardiovascular risk factors such as obesity and impaired glucose metabolism.1-7

Regular food intake plays a pivotal role in normal glucose homeostasis. Animal studies using knock-out mice have shown that hepatic insulin signaling via two kinds of insulin receptor substrate (IRS) works in a complicated manner in before and after feeding. ${ }^{8-10}$ However, the pathogenic feature of skipping breakfast, which theoretically aggravates the fasting condition in the morning, has not been addressed in a clinical study, particularly in terms of glucose metabolism. Furthermore, theoretically, skipping breakfast means a lack of supplementary energy and increased risk of hypoglycemia, which in turn stimulates eating behavior.

In this context, we examined the associations between skipping breakfast with impaired glucose metabolism along with cardiovascular risk factors, including circulating $\mathrm{C}$-reactive protein (CRP), a risk factor for type 2 diabetes, ${ }^{11,12}$ and lifestyle factors in a cross-sectional study of asymptomatic adults who underwent a detailed medical check-up. Impaired fasting glucose (IFG) and impaired glucose tolerance (IGT) are involved in the pathophysiology of impaired glucose homeostasis and represent the pre-diabetic state. ${ }^{13-15}$ Therefore, we further investigated the associations of skipping breakfast with these pre-diabetes conditions in a subgroup of subjects who underwent a $75 \mathrm{~g}$ oral glucose tolerance test (OGTT).

\section{Materials and Methods}

This study was based on a composite research program that was conducted in collaboration with Josai University, Sakado, Japan, and the Social Insurance Omiya General Hospital, Saitama, Japan. The protocol was approved by the Ethics Committee of Josai University and the Ethics Committee of the Hospital. All subjects gave their informed consent.
Correspondence: Kei Nakajima, Division of Clinical Nutrition, Department of Medical Dietetics, Faculty of Pharmaceutical Sciences, Josai University, 1-1 Keyakidai, Sakado, Saitama, 350-0295, Japan.

Tel. +81.49.271.7260 - Fax +81.49.271.7260.

E-mail: keinaka@josai.ac.jp

Key words: skipping breakfast, impaired fasting glucose , lifestyle factors, oral glucose tolerance test, C-reactive protein.

Conflict of interest: the authors have no conflicts of interest to disclose.

Contributions: KN and HM designed the study. $\mathrm{KN}$, YL, and TN collected and analyzed the data. $\mathrm{KN}, \mathrm{YL}, \mathrm{TN}, \mathrm{ST}, \mathrm{MS}$, and ME researched and evaluated the literature. $\mathrm{KN}$ wrote the first draft of the manuscript. All authors reviewed and edited the manuscript, and approved the final version for publication.

There was no personal or financial support or author involvement with organization(s) with financial interest for this study.

Received for publication: 27 September 2011. Revision received: 3 November 2011.

Accepted for publication: 3 November 2011

This work is licensed under a Creative Commons Attribution NonCommercial 3.0 License (CC BYNC 3.0)

(C) Copyright Y. Li et al., 2011

Licensee PAGEPress, Italy

Endocrinology Studies 2011; 1:e17

doi:10.4081/es.2011.e17

\section{Subjects}

We analyzed data collected from asymptomatic adults aged $30-80$ years who voluntarily underwent a detailed regular medical check-up between May 2008 and March 2010.

Subjects were required to complete a standard questionnaire now widely used in Japan. We initially recruited 2,448 subjects in this program. Subjects with self-reported use of insulin and/or oral medications for diabetes were excluded from the study. Subjects with elevated circulating CRP of $10.0 \mathrm{mg} / \mathrm{L}$ or more were also excluded because of latent inflammatory diseases. A total of 2,331 subjects (men $n=1,515$; women, $\mathrm{n}=816$ ) with completed self-report questionnaires and biochemical/anthropometric data were finally included in this study.

The questionnaire was developed by the Japanese Ministry of Health, Labour and Welfare in 2007, primarily to prevent metabolic syndrome and cardiovascular diseases through a better understanding of individual lifestyle factors. 16 The questionnaire is now widely used in Japan at regular mandatory medical check- 
ups for adults forty years old or over. The questionnaire consists of 22 questions, including eating behavior (e.g. skipping breakfast and late dinner before bedtime), physical activities (e.g. frequent or infrequent exercise), smoking (non-smoker or current smoker), habitual alcohol consumption (none, occasionally, daily), pharmacotherapy (e.g. for hypertension, hypercholesterolemia and diabetes) and past medical history (e.g. cardiovascular disease and stroke). The suspected habitual skipping breakfast (SHSB) was determined with a positive response to the simple question, Do you skip breakfast at least three times per week? The definitions of late dinner and infrequent exercise in the questionnaire were as follows: dinner within two hours before bedtime at least 3 times/week, and a session of more than $30 \mathrm{~min}$ exercise less than 2 times/week, respectively. All questions were to be answered dichotomously, either present'or absent.

\section{Laboratory measurements}

All biochemical variables were measured in the same laboratory using standard methods in samples obtained after an overnight fast. In this study, gastric empty was confirmed by upper gastrointestinal fluoroscopy or endoscopy in the check-up. Fasting plasma glucose (FPG) was measured by the glucose oxidase method. HbAlc was measured in Japan Diabetes Society (JDS)-HbAlc units by high-performance liquid chromatography. HbAlc was converted to National Glycohemoglobin Standardization Program (NGSP) levels by the formula HbAlc (\%) (NGSP)=HbAlc (JDS) (\%)+0.4\%, considering the relational expression of HbAlc (JDS) (\%) measured by the previous Japanese standard substance and measurement methods. ${ }^{17}$

\section{Definition of pre-diabetes}

Elevated blood pressure ( $\geq 130 / 85 \mathrm{mmHg}$ ), hypertriglyceridemia $(\geq 150 \mathrm{mg} / \mathrm{dL}$ ) and low high-density lipoprotein cholesterol (HDL-C) ( $<40 \mathrm{mg} / \mathrm{dL}$ for men, $<50 \mathrm{mg} / \mathrm{dL}$ for women) were defined according to the definition of metabolic syndrome by the National Cholesterol Education Program Expert Panel criteria. ${ }^{18}$ IFG (FPG 100-125 mg/dL) was defined according to the American Diabetes Association (ADA). ${ }^{19}$ When impaired glucose metabolism was assessed by FPG, subjects were divided into three groups according to the degree of FPG (normal, FPG: <100 mg/dL; IFG: 100-125 mg/dL; high, FPG $\geq 126 \mathrm{mg} / \mathrm{dL}$ ). When impaired glucose metabolism was assessed by HbAlc, subjects were divided into three groups according to the NGSP HbAlc (normal, <5.7\%; borderline, 5.7-
$6.4 \%$; diabetic, $\geq 6.5 \%$ ). Subjects with suspected pre-diabetes or diabetes, and subjects who wished to know their glucose metabolism in more detail, underwent an OGTT. Subjects with definite diabetes (under treatment consisting of diet and exercise) did not undergo an 0GTT. Consequently, 1,315 subjects (912 men and 403 women) underwent the test. Isolated IFG (IIFG) (FPG 100-125 mg/d and 2-hOGTT <140 $\mathrm{mg} / \mathrm{dL}$ ), isolated IGT (I-IGT) (FPG $<100 \mathrm{mg} / \mathrm{dL}$ and 2-h OGTT 140-199 $\mathrm{mg} / \mathrm{dL})$, combined IFG/IGT (FPG 100-125 mg/dL and 2-h OGTT 140$199 \mathrm{mg} / \mathrm{dL}$ ), diabetic (FPG $\geq 126 \mathrm{mg} / \mathrm{dL}$ or 2 -h OGTT $\geq 200 \mathrm{mg} / \mathrm{dL}$ ), and normal glucose tolerance (NGT) (FPG $<100 \mathrm{mg} / \mathrm{dL}$ and 2-hour OGTT $<140 \mathrm{mg} / \mathrm{dL}$ ) were defined according to ADA criteria. ${ }^{13}$ High and normal C-reactive protein (CRP) level was defined as levels above the $90^{\text {th }}$ percentile $(>1.8 \mathrm{mg} / \mathrm{L})$ and below the $75^{\text {th }}$ percentile $(<0.8 \mathrm{mg} / \mathrm{L})$, respectively.

\section{Statistical analysis}

Data are expressed as means \pm SD. Triglyceride and CRP levels are expressed as medians (interquartile range). Differences in clinical variables between the subjects who regularly skipped breakfast and those who did not were examined with analysis of covariance (ANCOVA) controlling for age. Multivariate logistical regression was used to examine the associations between skipping breakfast and impaired glucose metabolism, lifestyle factors, and clinical variables, and to calculate Odds Ratios (OR) and $95 \%$ confidence intervals (95\% CI) after adjustment for confounders. Statistical analysis was performed using IBM-SPSS version 18.0 (SPSS; Chicago, IL, USA) and Statview version 5.0 (SAS Institute; Cary, NC, USA). Values of $\mathrm{P}<0.05$ were considered statistically significant.

\section{Results}

The characteristics of subjects according to SHSB are shown in Table 1. The overall prevalence of SHSB was $16.3 \%$ (20.1\% for men and $9.4 \%$ for women, $\mathrm{P}<0.0001, \chi^{2}$ test). Subjects with SHSB were significantly younger (by approximately 8 years) than those without. The prevalence of men, current smoker, drinking alcohol everyday, late dinner, and infrequent exercise were significantly higher in those with SHSB, even after adjustment for age with logistical regression analysis (all $\mathrm{P}<0.0001)$. However, no significant difference in cardiovascular risk factors, except triglyceride, was found between subjects with SHSB and those without. Multiple logistic analyses showed that IFG, age (inversely), smoking, a late dinner, infrequent exercise and high CRP

Table 1. Characteristics of subjects according to suspected habitual skipping breakfast.

\begin{tabular}{|c|c|c|c|}
\hline & SHSB & Non-SHSB & P* \\
\hline n. & 381 & 1,950 & \\
\hline Age, $y$ & $46.9 \pm 10.0$ & $54.7 \pm 11.7$ & $<0.0001$ \\
\hline Men, n. (\%) & $304(79.8)$ & $1,211(62.1)$ & $<0.0001$ \\
\hline Body mass index, $\mathrm{kg} / \mathrm{m}^{2}$ & $23.7 \pm 3.6$ & $23.2 \pm 3.1$ & 0.04 \\
\hline Systolic blood pressure, $\mathrm{mmHg}$ & $119 \pm 17.9$ & $122 \pm 19.3$ & ns \\
\hline Diastolic blood pressure, $\mathrm{mmHg}$ & $75.3 \pm 12.8$ & $75.7 \pm 12.8$ & $\mathrm{~ns}$ \\
\hline Total cholesterol, mg/dL & $206 \pm 33.8$ & $206 \pm 35.9$ & ns \\
\hline Triglyceride, mg/dL & $109(75-157)$ & $92.5(66-134)$ & $<0.0001$ \\
\hline HDL-C, mg/dL & $59.1 \pm 15.5$ & $61.5 \pm 14.8$ & ns \\
\hline Fasting plasma glucose, mg/dL & $101 \pm 12.7$ & $99.3 \pm 15.7$ & ns \\
\hline HbAlc, $\%$ & $5.6 \pm 0.4$ & $5.7 \pm 0.5$ & ns \\
\hline C-reactive protein, mg/L & $0.50(0.30-1.00)$ & $0.40(0.30-0.80)$ & ns \\
\hline $\begin{array}{l}\text { Prior history } \\
\text { Cardiovascular disease, n. (\%) } \\
\text { Stroke, n. (\%) }\end{array}$ & $\begin{array}{l}6(1.6) \\
2(0.5)\end{array}$ & $\begin{array}{l}70(3.6) \\
37(1.8)\end{array}$ & $\begin{array}{l}\text { ns } \\
\text { ns }\end{array}$ \\
\hline $\begin{array}{l}\text { Medications } \\
\text { Hypertension, n. (\%) } \\
\text { Hypercholesterolemia, n. (\%) }\end{array}$ & $\begin{array}{l}35(9.2) \\
18(4.7)\end{array}$ & $\begin{array}{l}343(17.6) \\
207(10.6)\end{array}$ & $\begin{array}{l}\text { ns } \\
\text { ns }\end{array}$ \\
\hline Current smoker, n. (\%) & $183(48.0)$ & $429(22.0)$ & $<0.0001$ \\
\hline Drinking alcohol every day, n. (\%) & $149(39.1)$ & $585(30.0)$ & $<0.0001$ \\
\hline Late dinner, n. (\%) & $224(58.8)$ & $549(28.2)$ & $<0.0001$ \\
\hline Infrequent exercise, n. (\%) & $321(84.3)$ & $1,283(65.8)$ & $<0.0001$ \\
\hline
\end{tabular}

Data are expressed as means $\pm \mathrm{SD}$ or $\mathrm{n}(\%)$. Triglyceride and C-reactive protein are expressed as medians (interquartile range). ${ }^{*} \mathrm{P}$ was examined with ANCOVA controlling for age. Triglyceride and C-reactive protein were analyzed after log transformation. P in prevalence of clinical factors were examined with logistical regression analysis after adjustment for age. SHSB, suspected habitual skipping breakfast; HDL-C, highdensity lipoprotein cholesterol. 
were significantly and independently associated with SHSB, even after adjustment for residual confounders (Table 2). Further adjustment for continuous $\mathrm{HbAlc}$ strengthened the association between IFG and SHSB (Model 5).

However, body mass index (BMI), elevated blood pressure, and dyslipidemia were not significantly associated with SHSB after adjustment for confounders, particularly age and sex, and neither was waist circumference (data not shown). Of note, when late dinner was considered as a dependent variable, IFG was not significantly associated with late dinner, per se, after adjustment for residual confounders, particularly drinking alcohol [1.20 (0.98-1.48), after adjustment for age, sex, and drinking alcohol, data not shown]. In contrast, when impaired glucose metabolism was evalu- ated with HbAlc (Table 3), borderline and diabetic HbAlc levels were not significantly associated with SHSB after adjustment for confounders, although overall FPG was highly correlated with $\mathrm{HbAlc}$ in this study as generally expected $(\mathrm{r}=0.77, \mathrm{P}<0.0001$, Pearson correlation, data not shown). However, age, smoking, late dinner, infrequent exercise, and high CRP were significantly associated with SHSB in multivariate models, as in Table 2. There was no significant association of Borderline and Diabetic HbAlc levels with SHSB after adjustment for confounders, although overall FPG was highly correlated with HbAlc in this study, as generally expected $(\mathrm{r}=0.77, \mathrm{P}<0.0001$, Pearson's correlation, data not shown). However, age, smoking, late dinner, infrequent exercise, and high CRP were signifi- cantly associated with SHSB in multivariate models, as in Table 2.

As shown in Table 4, as compared with NGT, I-IFG, but not the other categories of OGTT results, was significantly associated with SHSB. This association remained after adjustment for residual confounders.

It is worthy of note that when subjects with a past history of heart disease and stroke, as well as those treated with medications, were excluded from the study and the same analysis was conducted, similar results were obtained; SHSB was significantly associated with IFG but not IGT, even after adjustment for the same confounders as in Table 2: $\mathrm{n}=1,793,1.69$ (1.27-2.24), $\mathrm{P}=0.0003$ and 1.25 (0.58-2.69), $\mathrm{P}=0.57$, respectively (data not shown).

Table 2. Associations between the degree of fasting plasma glucose and confounding factors with suspected habitual skipping breakfast.

$\begin{array}{lllll}\text { Model } 1 & \text { Model } 2 & \text { Model 3 } & \text { Model } 5\end{array}$

FPG

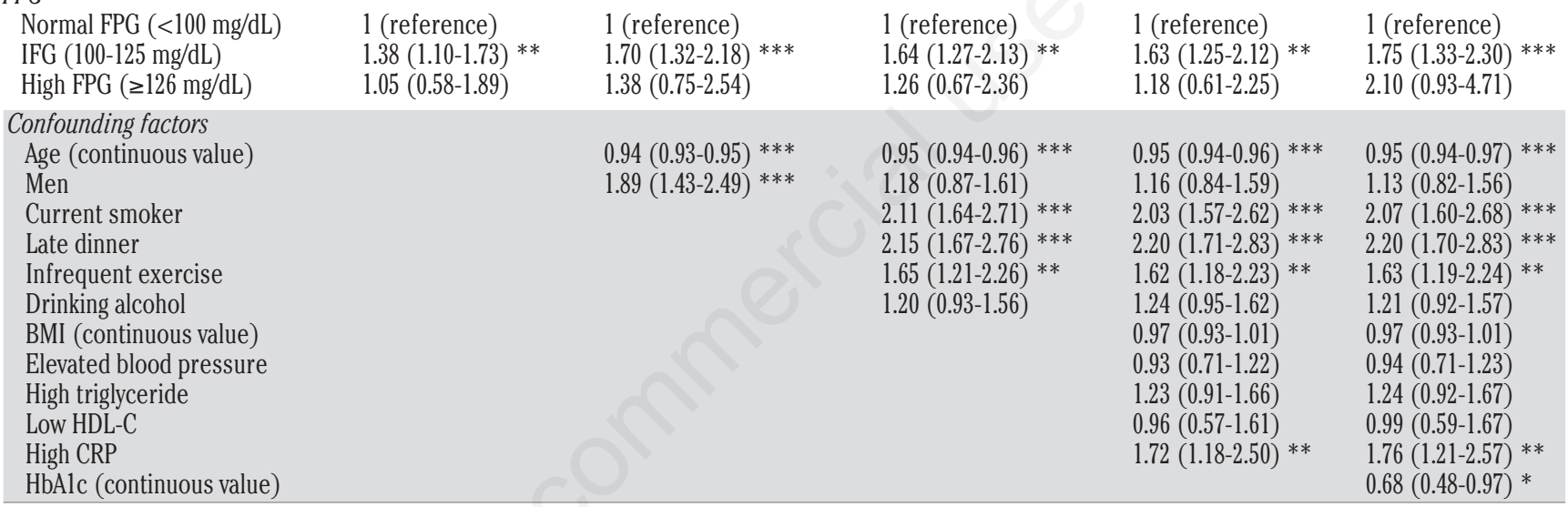

The Normal FPG, IFG, and High FPG included 1425, 815, and 91 subjects, respectively. Model 1: unadjusted; Model 2: adjusted for age and sex (men); Model 3: Model 2 plus adjustment for current smoking ( $v$ s nonsmokers), late dinner ( $v s$ non-late dinner), infrequent exercise ( $v s$ frequent exercise), drinking alcohol every day (vs infrequent/no alcohol consumption); Model 4: Model 3 plus adjustment for BMI, elevated blood pressure, dyslipidemia, and high CRP ( $>90^{\text {th }}$ percentile [ $\left.>1.8 \mathrm{mg} / \mathrm{L}\right]$ vs $<75^{\text {th }}$ percentile $[<0.8 \mathrm{mg} / \mathrm{L}]$ ); Model 5 : Model 4 plus adjustment for HbAlc.SHSB, suspected habitual skipping breakfast; FPG, fasting plasma glucose; BMI, body mass index; HDL-C, high-density lipoprotein cholesterol; CRP, C-reactive protein; ${ }^{*} \mathrm{P}<0.05,{ }^{* *} \mathrm{P}<0.01,{ }^{* * * \mathrm{P}<0.0001}$.

Table 3. Associations between the degree of HbAlc and confounding factors with suspected habitual skipping breakfast.

\begin{tabular}{|c|c|c|c|c|c|}
\hline & Model 1 & Model 2 & Model 3 & Model 4 & Model 5 \\
\hline \multicolumn{6}{|l|}{ HbAlc } \\
\hline Normal $<5.7 \%$ & 1 (reference) & 1 (reference) & 1 (reference) & 1 (reference) & 1 (reference) \\
\hline Borderline 5.7-6.4\% & $0.58(0.46-0.74) * * *$ & $0.99(0.76-1.28)$ & $0.95(0.73-1.24)$ & $0.89(0.67-1.17)$ & $0.81(0.61-1.08)$ \\
\hline Diabetic $\geq 6.5 \%$ & $0.66(0.36-1.20)$ & $1.03(0.55-1.92)$ & $0.96(0.50-1.83)$ & $0.82(0.42-1.62)$ & $0.38(0.15-1.00)$ \\
\hline \multicolumn{6}{|l|}{ Confounding factors } \\
\hline Age (continuous value) & & $0.94(0.93-0.96) * * *$ & $0.96(0.95-0.97) * * *$ & $0.96(0.95-0.97) * * *$ & $0.96(0.94-0.97) * * *$ \\
\hline Men & & $2.08(1.58-2.73) * * *$ & $1.29(0.95-1.74)$ & $1.22(0.89-1.68)$ & $1.17(0.85-1.61)$ \\
\hline Current smoker & & & $2.07(1.61-2.67) * * *$ & $1.99(1.54-2.57) * * *$ & $2.00(1.54-2.58) * * *$ \\
\hline Late dinner & & & $2.17(1.69-2.78) * * *$ & $2.22(1.72-2.85) * * *$ & $2.21(1.71-2.84)$ *** \\
\hline Infrequent exercise & & & $1.67(1.22-2.28) * *$ & $1.63(1.19-2.23) * *$ & $1.61(1.17-2.21) * *$ \\
\hline Drinking alcohol & & & $1.24(0.96-1.61)$ & $1.29(0.99-1.68)$ & $1.26(0.96-1.64)$ \\
\hline BMI (continuous value) & & & & $0.98(0.94-1.02)$ & $0.97(0.93-1.02)$ \\
\hline Elevated blood pressure & & & & $0.98(0.75-1.29)$ & $0.96(0.73-1.26)$ \\
\hline High triglyceride & & & & $1.27(0.94-1.72)$ & $1.27(0.94-1.71)$ \\
\hline Low HDL-C & & & & $0.95(0.56-1.60)$ & $0.98(0.58-1.66)$ \\
\hline High CRP & & & & $1.76(1.21-2.57) * *$ & $1.75(1.20-2.55)$ ** \\
\hline FPG (continuous value) & & & & & $1.01(1.00-1.02) *$ \\
\hline
\end{tabular}

The Normal HbAlc, Borderline HbAlc, and Diabetic HbAlc included 1239, 997, and 94 subjects, respectively. Models 1-4 are the same as those used in Table 2, Model 5: Model 4 plus adjustment for FPG. SHSB, suspected habitual skipping breakfast; FPG, fasting plasma glucose; BMI, body mass index; HDL-C, high-density lipoprotein cholesterol; CRP, C-reactive protein; ${ }^{* P}<0.05,{ }^{* *} \mathrm{P}<0.01,{ }^{* * *} \mathrm{P}<0.0001$. 


\section{Discussion}

This pilot study was primarily designed to explore a possible association of habitual skipping breakfast, roughly assessed with a simple question, with IFG in asymptomatic adults, who were mostly non-obese not undergoing treatment for diabetes. A National Health and Nutrition Survey in Japan 2009 reported that the prevalence of skipping breakfast was $11.6 \%$ in the general population and over $20 \%$ in people aged $20-39$ years,${ }^{20}$ which is consistent with current results. In addition, elevated circulating CRP and some unfavorable lifestyle factors were also independently associated with SHSB. In contrast, none of the components of metabolic syndrome were associated with skipping breakfast. It is worthy of note that, of pre-diabetes conditions, SHSB was only associated with IIFG, compared to NGT. A significant association between SHSB and a late dinner suggests the possibility of a nocturnal life-style in subjects with a habit of skipping breakfast, ${ }^{21,22}$ rather than intentionally skipping breakfast as part of a diet. Indeed, a late dinner before sleeping may impair postprandial glucose metabolism and food digestion, and may lead to prolonged elevated FPG and poor appetite in the morning. Therefore, in some subjects who skip breakfast, the physical condition in the early morning might be close to prolonged postprandial state rather than distinct fasting state. However, a late dinner is unlikely to be a major factor relating to IFG because a late dinner was not associated with IFG after controlling for drinking alcohol and other confounders. Furthermore, the absence of an association between SHSB with IGT, and the inverse association between SHSB and diabetic glucose profiles, including findings assessed with HbAlc, suggest that overall, hyperglycemia is not observed in individuals who regularly skip breakfast.

Alternatively, a significant association of SHSB with IFG, but not high FPG, may suggest a possibility of reverse causality because subjects with a past history of heart disease and stroke, as well as those treated with medications, are likely to change their dietary habits for health reasons. However, when such subjects were excluded from the data, similar results were obtained, suggesting the unlikelihood of reverse causality. Recently, Smith et al. ${ }^{\text {? }}$ have published a prospective study reporting that people who regularly skipped breakfast in childhood and adulthood had significantly higher fasting glucose and higher fasting insulin over a 20-year period compared with those who did not, and their findings appear to be consistent with our results. However, further evaluation of the associations with impaired glucose metabolism, such as elevated HbAlc and IGT, was not made in their study. There is evidence that peripheral (muscle) insulin resistance is a major pathogenic feature in individuals with isolated IGT,15 whereas that of isolated IFG is rather controversial. Kim et al. ${ }^{23}$ reported that peripheral insulin resistance varied considerably in individuals with IFG, with the conclusion that the majority of IFG individuals are insulin resistant and the minority are insulin sensitive. This suggests a possible heterogeneity in insulin sensitivity in IFG. Nevertheless, several studies have shown that IFG is less strongly associated with future risk of type 2 diabetes, ${ }^{24}$ peripheral insulin resistance, ${ }^{25,26}$ and cardiovascular diseases, ${ }^{13-15}$ as compared with IGT. A plausible reason for these discrepancies may be the use of surrogate markers, such as homeostasis model assessment-insulin resistance, rather than direct measurements of insulin resistance as discussed by Kim et al. ${ }^{23}$ or discordance in the definition of IFG. In this study, infrequent exercise, smoking, and elevated CRP,11,12 all of which are related with insulin resistance, but not with any of the components of metabolic syndrome, were observed in subjects with SHSB. This apparently curious association might result in a possible heterogeneity in insulin sensitivity in IFG. Meanwhile, people with IFG predominantly exhibit hepatic insulin resistance, ${ }^{15,25-27}$ which increases fasting hepatic glucose production and may account for elevated FPG in the morning and for skipping breakfast because the subject is not hungry. Bock et al. ${ }^{27}$ reported that individuals with IFG had hepatic insulin resistance because of impaired insulin-mediated suppression of endogenous glucose production during the fasting state. Nevertheless, endogenous glucose production is rapidly suppressed by the rising insulin concentration immediately after eating because the second-phase insulin response is not attenuated. ${ }^{27,28}$ Kubota et al. ${ }^{8}$ proposed the concept of the existence of a functional relay between IRS-1 and IRS-2 in hepatic insulin action during fasting and re-feeding from the results of their study using IRS knock-out mice. Briefly, IRS-1 functions primarily after re-feeding, whereas IRS-2 functions mainly during fasting and immediately after the start of refeeding. Considering that fasting glucose levels were slightly increased in Irs2 knock-out mice in the study by Dong et al., ${ }^{9}$ it is possible that IRS-2 might be attenuated in individuals with IFG. Thus, as Haeusler et al.10 proposed, IFG should be re-examined in terms of emerging findings concerning insulin signaling via IRS. Along these lines, IFG should also be considered in terms of skipping breakfast because this habit theoretically aggravates the fasting state further.

Meanwhile, social factors may also play a role in the associations observed here. For example, the meal times of individuals are often determined by social factors rather than biological need, $3,6,29$ indicating that the habit of skipping breakfast may be an unintentional, unfavorable lifestyle factor related to individual circumstances and other factors not examined here, such as the intake of caffeine, snacks, beverages and nutrients. To fully address the underlying mechanisms, future studies should examine the associations between social factors and food intake.

Many previous studies, ${ }^{1-3,6}$ but not all, $, 5,5,7,30$ have reported that skipping breakfast predisposes towards being overweight and obesity. Indeed, in this study, SHSB was significantly associated with overweight/obesity $(\mathrm{BMI} \geq 25.0$ $\mathrm{kg} / \mathrm{m}^{2}$ ) before controlling for confounders [1.42 (1.12-1.80), $\mathrm{P}=0.004$ ]. However, this association disappeared after adjustment for gender [ 1.23 (0.96-1.57), $\mathrm{P}=0.10$ ] and after full adjustment for all confounders in Table 2 . In the analysis of data from NHANES III, ${ }^{31}$ an inverse association between breakfast consumption and a lower body mass index was observed in women, but not in men. This is partially consistent with our results because there were more men (65\%) than women in this study. Alternatively, the small number of obese subjects $\left(\mathrm{BMI} \geq 30 \mathrm{~kg} / \mathrm{m}^{2}, 3.3 \%\right.$ of total subjects) may

Table 4. Associations between FPG/2 h OGTT profiles and suspected habitual skipping breakfast.

\begin{tabular}{|c|c|c|c|c|}
\hline & Model 1 & Model 2 & Model 3 & Model 4 \\
\hline \multicolumn{5}{|l|}{ FPG/2-h OGTT profile } \\
\hline NTG & 1 (reference) & 1 (reference) & 1 (reference) & 1 (reference) \\
\hline I-IFG & $1.62(1.15-2.28) *$ & $1.96(1.35-2.84) * *$ & $1.94(1.32-2.85) * *$ & $1.87(1.26-2.77) *$ \\
\hline I-IGT & $0.61(0.33-1.13)$ & $0.76(0.41-1.43)$ & $0.77(0.40-1.48)$ & $0.68(0.35-1.33)$ \\
\hline Combined I-IFG/I-IGT & $1.01(0.63-1.62)$ & $1.37(0.83-2.26)$ & $1.53(0.91-2.57)$ & $1.37(0.80-2.35)$ \\
\hline Diabetic & $0.96(0.57-1.62)$ & $1.41(0.81-2.46)$ & $1.41(0.79-2.49)$ & $1.34(0.73-2.48)$ \\
\hline
\end{tabular}


explain the inconsistency with previous studies. 4,30 This study has some limitations. First, because of its cross-sectional design, we cannot determine causality or whether SHSB was a result (unintentional) or a cause (intentional) of the factors identified in regression models. Some subjects with SHSB likely skipped breakfast to lose weight (e.g. on a diet). Well designed prospective studies are needed to confirm the current findings and to clarify the underlying mechanism in more detail. Second, the quantity, type and timing of food intake were not recorded because the study was not originally conducted to explore the relationship between skipping breakfast and metabolic abnormalities. Therefore, we could not take into account the amount or the content of the meals consumed each day. A detailed assessment of food intake by food-frequency questionnaires, for example, will help us to further explore the interactions between skipping breakfast and impaired glucose metabolism. Third, SHSB was defined as a dichotomous (binary) variable as at least 3 times/week versus another, using a standard questionnaire. We were not, therefore, able to examine linear associations. To achieve this, the questionnaires should record intermediate categories, such as occasionally skipping breakfast. However, adjustment for late dinner, a similarly undesirable eating behavior, and other lifestyle factors may attenuate the bias provided by the binary assessment. Finally, because this study included mostly non-obese individuals without diabetes, the findings may not be applicable to those with diabetes or cardiovascular diseases.

In conclusion, our findings suggest a possible association between SHSB and IFG, but not with any of the components of metabolic syndrome, in asymptomatic adults. Furthermore, subtle inflammatory state (high CRP) and some unfavorable lifestyle factors (e.g. smoking), which are potent cardiovascular and pre-diabetic state risk factors, were also associated with SHSB. These findings, the causality, and the clinical relevance need to be confirmed in future, prefereably prospective studies.

\section{References}

1. Hoyland A, Dye L, Lawton CL. A systematic review of the effect of breakfast on the cognitive performance of children and adolescents. Nutr Res Rev 2009;22:220-43.

2. Keski-Rahkonen A, Kaprio J, Rissanen A, et al. Breakfast skipping and health-compromising behaviors in adolescents and adults. Eur J Clin Nutr 2003;57:842-53.

3. Berkey CS, Rockett HR, Gillman MW, et al. Longitudinal study of skipping breakfast and weight change in adolescents. Int $\mathrm{J}$ Obes Relat Metab Disord 2003;27:1258-66.
4. Timlin MT, Pereira MA. Breakfast frequency and quality in the etiology of adult obesity and chronic diseases. Nutr Rev 2007;65:26881.

5. Nishiyama M, Muto T, Minakawa T, et al. The combined unhealthy behaviors of breakfast skipping and smoking are associated with the prevalence of diabetes mellitus. Tohoku J Exp Med 2009;218:259-64.

6. Huang CJ, Hu HT, Fan YC, et al. Associations of breakfast skipping with obesity and health-related quality of life: evidence from a national survey in Taiwan. Int $\mathrm{J}$ Obes 2010;34:720-5.

7. Smith KJ, Gall SL, McNaughton SA, et al. Skipping breakfast: longitudinal associations with cardiometabolic risk factors in the Childhood Determinants of Adult Health Study. Am J Clin Nutr 2010;93:1316-25.

8. Kubota N, Kubota T, Itoh S, et al. Dynamic functional relay between insulin receptor substrate 1 and 2 in hepatic insulin signaling during fasting and feeding. Cell Metab 2008;8:49-64.

9. Dong XC, Copps KD, Guo S, et al. Inactivation of hepatic Foxol by insulin signaling is required for adaptive nutrient homeostasis and endocrine growth regulation. Cell Metab 2008;8:65-76.

10. Haeusler RA, Accili D. The double life of Irs. Cell Metab 2008;8:7-9.

11. Ndumele CE, Pradhan AD, Ridker PM. Interrelationships between inflammation, C-reactive protein, and insulin resistance. $\mathrm{J}$ Cardiometab Syndr 2006;1:190-6.

12. Haffner SM. Pre-diabetes, insulin resistance, inflammation and CVD risk. Diabetes Res Clin Pract 2003;61:S9-S18.

13. Unwin N, Shaw J, Zimmet P, et al. Impaired glucose tolerance and impaired fasting glycaemia: the current status on definition and intervention. Diabet Med 2002;19:708-23.

14. Petersen JL, McGuire DK. Impaired glucose tolerance and impaired fasting glucose--a review of diagnosis, clinical implications and management. Diab Vasc Dis Res 2005;2:9-15.

15. Nathan DM, Davidson MB, DeFronzo RA, et al. Impaired fasting glucose and impaired glucose tolerance: implications for care. Diabetes Care 2007;30:753-9.

16. Ministry of Health, Labour and Welfare. [Standard health examination and guidance program.] Tokyo, 2007.

17. The Committee of Japan Diabetes Society on the diagnostic criteria of diabetes mellitus. Report of the Committee on the classification and diagnostic criteria of diabetes mellitus. J. Jpn Diabetes Soc 2010: 53: 45067.

18. Grundy SM, Cleeman JI, Daniels SR, et al. Diagnosis and management of the metabolic syndrome: an American Heart Association/National Heart, Lung, and Blood
Institute Scientific Statement. Circulation 2005;112:2735-52.

19. Genuth S, Alberti KG, Bennett P, et al. Follow-up report on the diagnosis of diabetes mellitus. Diabetes Care 2003;26: 31607.

20. Ministry of Health, Labour and Welfare. [National Health and Nutrition Survey in Japan 2009].

21. Samuelson G. Dietary habits and nutritional status in adolescents over Europe. An overview of current studies in the Nordic countries. Eur J Clin Nutr 2000;54: S21-8.

22. Arakawa M, Taira K, Tanaka H, et al. A survey of junior high school students' sleep habit and lifestyle in Okinawa. Psychiatry Clin Neurosci 2001;55:211-2.

23. Kim SH, Reaven GM. Isolated impaired fasting glucose and peripheral insulin sensitivity: not a simple relationship. Diabetes Care 2008;31:347-52.

24. Rasmussen SS, Glümer C, Sandbaek A, et al. Determinants of progression from impaired fasting glucose and impaired glucose tolerance to diabetes in a high-risk screened population: 3 year follow-up in the ADDITION study, Denmark. Diabetologia 2008;51:249-57.

25. Abdul-Ghani MA, Tripathy D, DeFronzo RA. Contributions of beta-cell dysfunction and insulin resistance to the pathogenesis of impaired glucose tolerance and impaired fasting glucose. Diabetes Care 2006;29: 1130-9.

26. Cali' AM, Bonadonna RC, Trombetta M, et al. Metabolic abnormalities underlying the different prediabetic phenotypes in obese adolescents. J Clin Endocrinol Metab 2008; 93:1767-73.

27. Bock G, Chittilapilly E, Basu R, et al. Contribution of hepatic and extrahepatic insulin resistance to the pathogenesis of impaired fasting glucose: role of increased rates of gluconeogenesis. Diabetes 2007; 56:1703-11.

28. Meyer C, Pimenta W, Woerle HJ, et al. Different mechanisms for impaired fasting glucose and impaired postprandial glucose tolerance in humans. Diabetes Care 2006;29:1909-14.

29. Waterhouse J, Minors D, Atkinson G, et al. Chronobiology and meal times: internal and external factors. Br J Nutr 1997;77: S29-38.

30. Dialektakou KD, Vranas PB. Breakfast skipping and body mass index among adolescents in Greece: whether an association exists depends on how breakfast skipping is defined. J Am Diet Assoc 2008;108:1517-25.

31. Song WO, Chun OK, Obayashi S, et al. Is consumption of breakfast associated with body mass index in US adults? J Am Diet Assoc 2005;105:1373-82. 